\title{
Levantamento e avaliação dos casos de leishmaniose visceral (calazar) no Município de Formosa do Rio Preto, Bahia, Brasil
}

\section{Edineuza Azevedo Costa ${ }^{1}$, Marcília Martins da Silva², Tancio Gutier Ailan Costa ${ }^{3}$}

${ }^{1}$ Curso de Tecnologia em Gestão Ambiental. Instituto Federal do Piauí. Campus Corrente. Rua Projetada Trinta e Seis, 380. Nova Corrente. Corrente-PI, Brasil (CEP 64980-000).

${ }^{2}$ Instituto Federal do Piauí. Campus Corrente. Rua Projetada Trinta e Seis, 380. Nova Corrente. Corrente-PI, Brasil (CEP 64980-000)

${ }^{3}$ Universidade Federal do Ceará. Mestrado em Ciência do Solo. Campus Pici. Fortaleza-CE (CEP 60455-900). E-mail: gutierailan@gmail.com.

Resumo. As últimas décadas foram marcadas por grandes surtos de doenças vinculadas às más condições de vida em espaços urbanos, na maioria dos casos decorrentes da transmissão por mosquitos vetores, apontados como os principais causadores de doenças urbanas, como é o caso da leishmaniose visceral. Esta é uma doença infecto-parasitária, popularmente conhecida como calazar, que pode acometer tanto humanos quanto animais, principalmente os canídeos. Além disso, apresenta elevado potencial de mortalidade e ampla distribuição geográfica. Neste sentido, o presente estudo teve como objetivo quantificar a ocorrência de casos notificados de leishmaniose visceral em humanos e animais no Município de Formosa do Rio Preto (Estado da Bahia), através da obtenção de dados de ocorrência no período de 2010 a 2016, junto à Secretaria Municipal de Saúde, levantamento bibliográfico, em nível nacional, estadual e municipal. A partir dos dados obtidos, calculou-se as taxas de incidência para os casos notificados em humanos. 0 estudo constatou que no período avaliado o município apresentou um total de oito casos de leishmaniose, notificados para humanos, e 360 para cães. Assim, a distribuição dos casos entre 2010 e 2016 apontou que o Município de Formosa do Rio Preto apresenta um pequeno histórico de casos confirmados da doença, sendo os maiores registros entre 2014 e 2015.

Palavras-chave: Leishmaniose humana; Doença infecciosa; Saúde pública.

Abstract. Survey and evaluation of visceral leishmaniasis (kala-azar) cases in the Municipality of Formosa do Rio Preto, Bahia State, Brazil. The last decades have been marked by large outbreaks of diseases related to bad living conditions in urban areas, most of them resulting from the transmission by vector
Recebido: $14 / 02 / 2018$

Aceito: $06 / 04 / 2018$

Publicado: $30 / 04 / 2018$

Acesso aberto

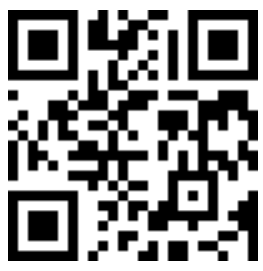

ORCID

() 0000-0002-8198-021X Edineuza Azevedo Costa

(1) 0000-0002-4906-7965 Marcília Martins da Silva

(1) 0000-0002-0635-2651 Tancio Gutier Ailan Costa 
mosquitoes, which are the main factor responsible for the transmission of urban diseases, such as visceral leishmaniasis. This is an infectious-parasitic disease, popularly known as kalaazar, which can affect both humans and animals, especially canids. In addition, it has a high mortality potential and wide geographic distribution. In this sense, this study aimed to quantify the occurrence of visceral leishmaniasis cases reported in humans and animals in the Municipality of Formosa do Rio Preto (Bahia State, Brazil), by obtaining data of occurrence, from 2010 to 2016, from the Municipal Health Department, carrying out a bibliographical survey, at the national, state and municipal levels. From the data obtained in the survey, the incidence rates of cases reported in humans were calculated. This study evidenced that, in the studied municipality, there were a total of eight cases of leishmaniasis in humans and 360 cases in dogs, in the evaluation period. Thus, the distribution of cases between 2010 and 2016 indicated that the Municipality of Formosa do Rio Preto has a small history of confirmed cases of visceral leishmaniasis and that most of the cases were recorded between 2014 and 2015.

Keywords: Human leishmaniasis; Infectious disease; Public health.

\section{Introdução}

As últimas décadas foram marcadas por grandes surtos de doenças vinculadas às más condições de vida em espaços urbanos, promovendo uma elevação expressiva nos casos de adoecimento das populações mundiais. Estes surtos, na maioria dos casos, são decorrentes das transmissões feitas por mosquitos vetores, apontados como os principais causadores de doenças urbanas nos últimos anos, como é o caso da leishmaniose visceral (LV).

A LV é uma zoonose cosmopolita, à exceção da Oceania. Na Índia, era conhecida como uma doença infecciosa de alta letalidade e denominada de calazar (doença negra), pela pigmentação escurecida na pele das pessoas acometidas pela infecção sistêmica, causada por um protozoário do gênero Leishmania (Gusmão et al., 2014).

Estudos revelam, através de dados registrados na América Latina, que 90\% dos casos acontecem no Brasil, onde a leishmaniose visceral humana (LVH) está entre as mais importantes doenças negligenciadas (Bonney, 2016), por ser grave, potencialmente fatal e cuja letalidade pode atingir $10 \%$, se o tratamento não for efetuado de forma adequada (Barbosa, 2013).

Segundo Gusmão et al. (2014), nos últimos anos o Brasil enfrenta a expansão da $\mathrm{LV}$ principalmente em áreas urbanas, apresentando casos positivos tanto em humanos quanto em animais, acometidos em várias cidades de pequeno, médio e grande porte. Assim, a ocupação desordenada dos centros urbanos em condições precárias de vida e em estado de destruição ambiental favorecem o desenvolvimento do vetor. Nestas condições, o vetor Lutzomyia longipalpisse adapta-se com facilidade às condições dos peridomicílios ricos em matéria orgânica gerado por animais domésticos e más condições sanitárias, passando a distribuir amplamente pelo país.

A doença é considerada uma enfermidade de grande importância para saúde pública, pois é responsável anualmente por 59.000 óbitos (Silva et al., 2010), resultante de aproximada- 
mente 500.000 casos da doença, com uma estimativa de 12 milhões de pessoas infectadas anualmente (OMS, 2012). Além disso, dentre os aproximadamente 42.067 registros de doentes nos últimos 12 anos no Brasil, 2.704 casos foram procedidos de óbitos, resultando em uma incidência média de 1,92 casos por 100.000 habitantes no país (Brasil, 2012).

De acordo com Montalvo et al. (2012), 90\% dos casos de infecção da zoonose ocorrem em países onde grande parte da população vivem em situação de extrema pobreza, a exemplo da ocorrência de casos no Brasil. Entretanto, não é apenas o fator financeiro um obstáculo para o controle e disseminação desta doença, pois além deste fator, as mudanças no comportamento humano também exercem significativa contribuição para aumento dos casos no país (Calvopina et al., 2004).

A evolução e aumento, nos últimos anos, dos casos de leishmaniose observados nas zonas de endemismo é resultado de falhas sistêmicas, relacionadas, sobretudo ao controle inadequado dos vetores e condições ambientes de transmissão, a crescente elevação dos casos da doença em pacientes imunodeprimidos, ao aumento da resistência do agente ao tratamento e ao impacto causado pelas mudanças climáticas, que contribuem para o incremento da transmissão da doença (Montalvo et al., 2012).

Além de humanos, os animais também são afetados pela leishmaniose por todo o mundo, entretanto sua predominância é observada na América do Sul e no Mediterrâneo, onde a leishmaniose está ampliando a sua área de ocorrência, atingindo locais onde ela era desconhecida. Pode-se citar a expansão da doença ao norte da Itália, nas províncias ao sul do Canadá (DantasTorres et al., 2012) no leste dos Estados Unidos (Petersen e Barr, 2009), no Equador (Calvopina et al., 2004). No
Brasil, a leishmaniose visceral é notificada na Bahia (Costa et al., 1988; Barboza et al., 2007), Ceará (Cavalcante et al., 2014; Rodrigues et al., 2017), Mato Grosso (Almeida et al., 2010), Mato Grosso do Sul (Marques et al., 2017), Minas Gerais (Silva et al., 2010; Gusmão et al., 2014), Paraíba (Silva et al., 2017), Pernambuco (Correia, 1998; Queiroz et al., 2004), Rio Grande do Norte (Ximenes et al., 2007; Barbosa, 2013; Leite e Araújo, 2013), Rio Grande do Sul (Souza et al., 2014) e São Paulo (Ortiz e Anversa, 2015).

Considerando esta problemática, a leishmaniose visceral permanece como um grave problema de saúde pública, de incidência elevada em vários municípios, principalmente na Região Nordeste do Brasil, com alta letalidade quando não diagnosticada precocemente, com alta demanda hospitalar e dos serviços públicos. Sendo assim, evidencia-se a importância de estudos para incentivar a tomada de decisão e controle da ocorrência de casos nos municípios nordestinos, inclusive os do oeste da Bahia. Deste modo, a leishmaniose representa um grande desafio tanto para os profissionais da saúde quanto para os representantes do governo.

Nesta perspectiva, estudos que abordem o padrão de ocorrência da leishmaniose visceral e suas características podem subsidiar $\mathrm{o}$ entendimento da dinâmica deste agravo no Brasil e, sobretudo, no Nordeste, contribuindo com ações importantes de vigilância em saúde e controle da doença (Barbosa, 2013). Assim, partindo da necessidade de levantamento de casos da doença na Região Nordeste de país, e especificamente no oeste da Bahia, o presente trabalho objetivou quantificar as ocorrências de casos notificados de leishmaniose visceral (calazar) em humanos e animais no Município de Formosa do Rio Preto, no oeste do Estado da Bahia. 


\section{Material e métodos}

\section{Área de estudo}

0 presente estudo foi desenvolvido no Município de Formosa do Rio Preto, localizado no extremo oeste do Estado da Bahia (latitude 11ㅇ 02' 54" S; longitude 45 11' 35" W; altitude de $490 \mathrm{~m}$ ). 0 município possui área de $16.185,171 \mathrm{~km}^{2}$, e uma população estimada de 25.652 habitantes (IBGE, 2016), e situa-se a $1.026 \mathrm{~km}$ da capital do estado, Salvador. 0 município é considerado o mais extenso pertencente ao estado, estando em 74을 lugar entre todos os municípios brasileiros, fazendo parte da Bacia do Rio Grande, importante afluente do Rio São Francisco.

O Município de Formosa do Rio Preto está localizado no domínio do Bioma Cerrado, com clima que possui duas estações bem definidas entre os períodos de chuva e estiagem. Desta forma, o clima é classificado como tropical subúmido e o período chuvoso está concentrado principalmente entre fevereiro e abril.

A região apresenta o volume de precipitações que varia entre 1.000 e $1.800 \mathrm{~mm}$ anuais e temperaturas médias anuais entre $25^{\circ} \mathrm{C}$ e $28^{\circ} \mathrm{C}$, e máximas na faixa de $30{ }^{\circ} \mathrm{C}$ a $33{ }^{\circ} \mathrm{C}$. De acordo com Ximenes (2007), as regiões com climas classificados como úmido ou subúmido e com temperaturas elevadas, como acontece com o Município de Formosa do Rio Preto, apresentam condições favoráveis para a proliferação de vetores de transmissão da leishmaniose visceral.

\section{Procedimentos metodológicos}

A pesquisa foi baseada numa revisão de literatura, através de levantamentos bibliográficos, documentais e utilização de pesquisas prévias sobre o assunto. No entanto, trata-se de um estudo descritivo, quantitativo e retrospectivo, que utilizou como fonte de informação os dados da Secretaria Municipal de Saúde e Vigilância Epidemiológica, do Estado da Bahia.
Foram analisadas informações sobre os casos de leishmaniose visceral humana e animais ocorridos no Município de Formosa do Rio Preto, no período de 2010 a 2016. A partir dos dados coletados, foram estimadas as taxas de incidência para cada ano de ocorrência da LV em humanos no município. As taxas foram calculadas segundo a metodologia proposta por Waldman (1998), com o auxílio da seguinte expressão matemática:

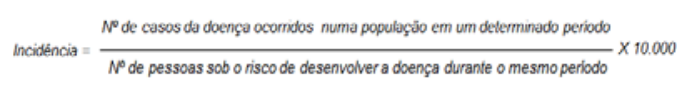

No cálculo de incidência da leishmaniose visceral foram utilizados dados populacionais para cada ano com ocorrência no município. Estes dados foram obtidos junto ao Instituto Brasileiro de Geografia e Estatística (IBGE), conforme projeção anual.

\section{Resultados e discussão}

\section{em humanos}

\section{Casos de leishmaniose visceral}

Foi verificado que no período de 2010 a 2016 o Município de Formosa do Rio Preto a notificação de oito casos da leishmaniose visceral (LV), notificados em humanos. Os casos diagnosticados e confirmados referem-se aos anos de 2015 e 2016, com ocorrência de 5 e 3 casos, respectivamente (Tabela 1). Para o período de 2010 a 2014, não foram confirmados casos da LV, entretanto foram registrados junto à Secretaria Municipal de Saúde 15 casos da doençaapontados como não reagentes.

Conforme demonstrado na Tabela 1, em 2015 o município apresentou o maior número de casos, comparado-se ao ano de 2016. Embora a diferença em números seja baixa, notouse que os casos em sua maioria foram referentes a indivíduos do sexo masculino, representando quatro casos para o ano de 2015, três casos para o ano de 2016, e apenas um caso para 
indivíduos do sexo feminino. Destes, apenas um caso refere-se a óbito no município.

Este resultado remete-se ao fato de que os indivíduos do sexo masculino são mais susceptíveis às parasitoses, como também pode estar relacionado com uma maior exposição a áreas de riscos ou até mesmo no desenvolver de atividades recreativas ou de trabalho, que envolva riscos de infecção se comparados aos indivíduos do sexo feminino.

Tabela 1. Ocorrência de casos de LV no Município de Formosa do Rio Preto, Bahia, no período de 2015 a 2016.

\begin{tabular}{|l|c|l|c|}
\hline Ano de ocorrência & Idade & Sexo & Bairro ou localidade \\
\hline 2015 & 8 anos & Masculino & Santa Helena \\
\hline 2015 & 59 anos & Masculino* & Centro \\
\hline 2015 & 7 anos & Masculino & Novo Horizonte \\
\hline 2015 & 48 anos & Masculino & Centro \\
\hline 2015 & 6 anos & Feminino & Santa Helena \\
\hline 2016 & 31 anos & Masculino & Zona Rural \\
\hline 2016 & 6 anos & Masculino & Centro \\
\hline 2016 & 39 anos & Masculino & Zona Rural \\
\hline
\end{tabular}

*Único caso de óbito ocorrido no município por conta da LV. Fonte: Secretaria Municipal de Saúde de Formosa do Rio Preto, Bahia. 2016.

WHO (2010) relata que, embora LV acometa indivíduos de ambos os sexos, indivíduos do sexo masculino são mais susceptíveis ao adoecimento por conta da doença e proporcionalmente mais afetado, baseado na existência de um fator hormonal ligado ao sexo, fatores comportamentais, como maior mobilidade em busca de atendimento médico em comparação com as mulheres, ou à presença destes indivíduos em áreas que oferecem maior risco de exposição aos vetores da doença como local de moradia, atividades laborais e/ou recreativas.

Quanto à faixa etária, a Tabela 1 mostra que $50 \%$ dos casos foram registrados em crianças entre 6 a 8 anos, sendo um do sexo feminino e os outros $50 \%$ em adultos entre 31e 59 anos de idade. Mesmo não havendo diferença numérica dos casos quanto à ocorrência em adultos e crianças, é possível constatar que além do crescimento de casos em crianças, a sua maioria pertencentes ao sexo masculino, bem como em adultos, podendo estar relacionado ao fato de que homens ao aumentarem sua idade passam a deixar uma maior área corporal exposta a picadas de vetores, visto que quase sempre permanecem sem camisa a maior parte do dia (Correia, 1998), principalmente quando estão no exercício de alguma atividade, seja ela de trabalho ou recreativa.

Em relação à distribuição espacial dos casos de LV no Município de Formosa do Rio Preto, pode-se constatar que nos dois anos com ocorrência da doença, o maior número de casos ocorreu nos habitantes do Bairro Centro, seguidos do Bairro Santa Helena e Novo Horizonte, situada na região norte da cidade e dois casos na zona rural, como mostra a Figura 2. 


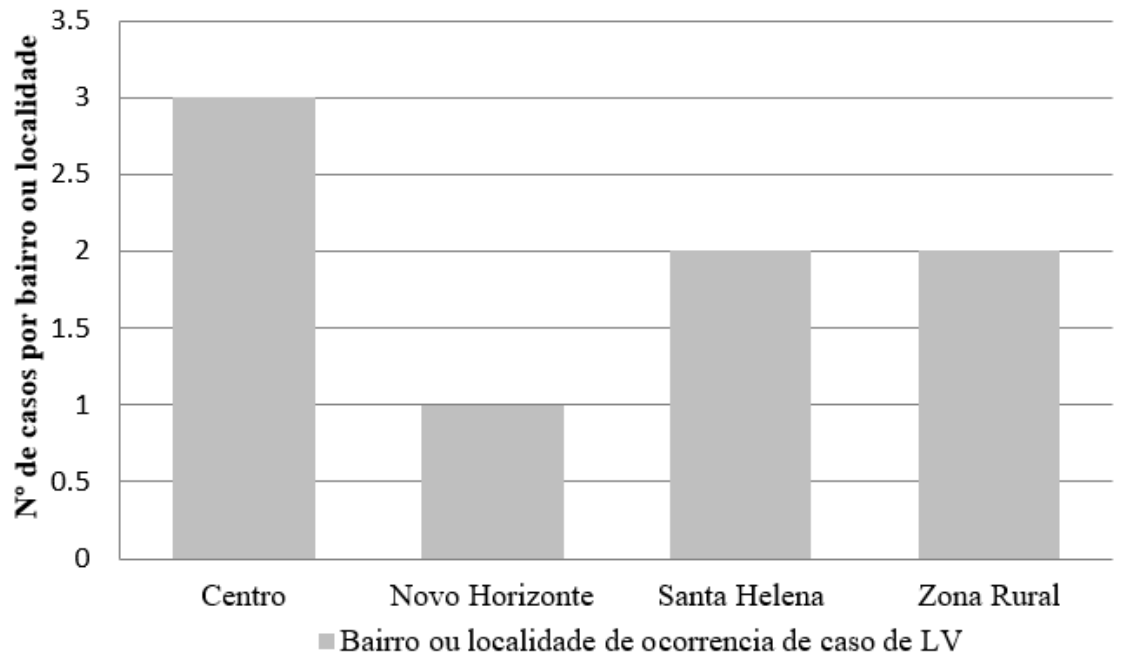

Figura 2. Distribuição dos casos de LV por bairro ou localidade do município de Formosa do Rio Preto, Bahia no período de 2015 a 2016.

Embora haja diferença quanto aos números de casos ocorrentes nos bairros do município e na zona rural, possivelmente em ambas as localidades os fatores relacionados à existência dos casos podem ser semelhantes. A ocorrência de casos no Bairro Centro pode estar relacionados com a existência de áreas naturais mais úmidas, como é o caso do Rio Preto e espaços naturais cobertos por vegetação, região limítrofe para os demais bairros da cidade, ambientes que são favoráveis à proliferação do vetor da LV. Além disso, como não foram observadas alterações climáticas consideráveis para o período de ocorrência de LV no município, a existência destes casos no perímetro urbano também pode ter relação com processo de transmissão através de cães, considerado o principal reservatório e o responsável pela persistência da doença em ambiente urbano.

Os casos na zona rural deve-se ao fato dos indivíduos estarem mais expostos aos ambientes de desenvolvimento do vetor, como é o caso de regiões de matas nativas (neste caso associado ao ambiente rural), onde o mosquito responsável pela transmissão mantem seu ciclo através dos reservatórios silvestres.

Houve um crescimento abrupto quanto à incidência da LV no município (Figura 3). Ao se observar os dados, percebe-se que de 2014 para 2015 houve um aumento direto dos casos da doença, apresentando uma taxa de aproximadamente 2/10.000 hab., fato que também aconteceu em 2016, visto que a diferença de incidência não foi tão significativa, se comparada com 2015, representado cerca de 1,16/10.000 hab. Ao comparamos a taxa de incidência calculada neste estudo com os dados publicados pela Secretaria de Saúde do Estado da Bahia - SESAB, observa-se que a taxa de incidência não ultrapassou os dados publicados referente ao ano de 2015 (Figura 4). 


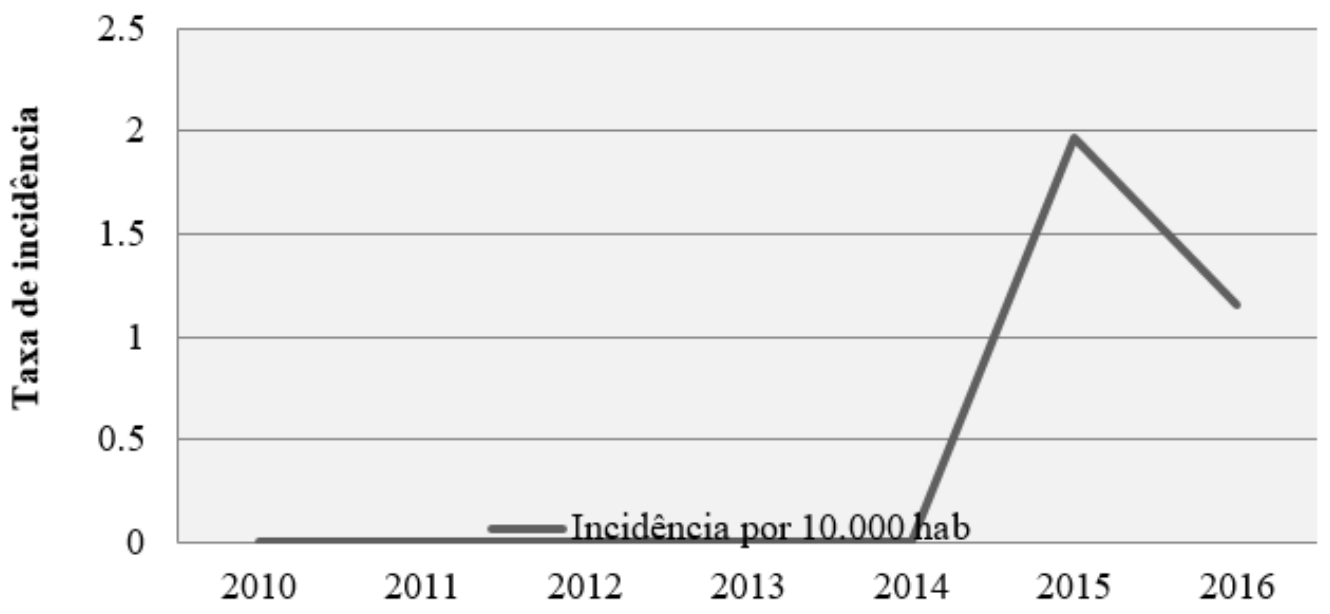

Figura 3. Taxa de incidência dos casos de LV do Município de Formosa do Rio Preto, Bahia, no período de 2010 a 2016.

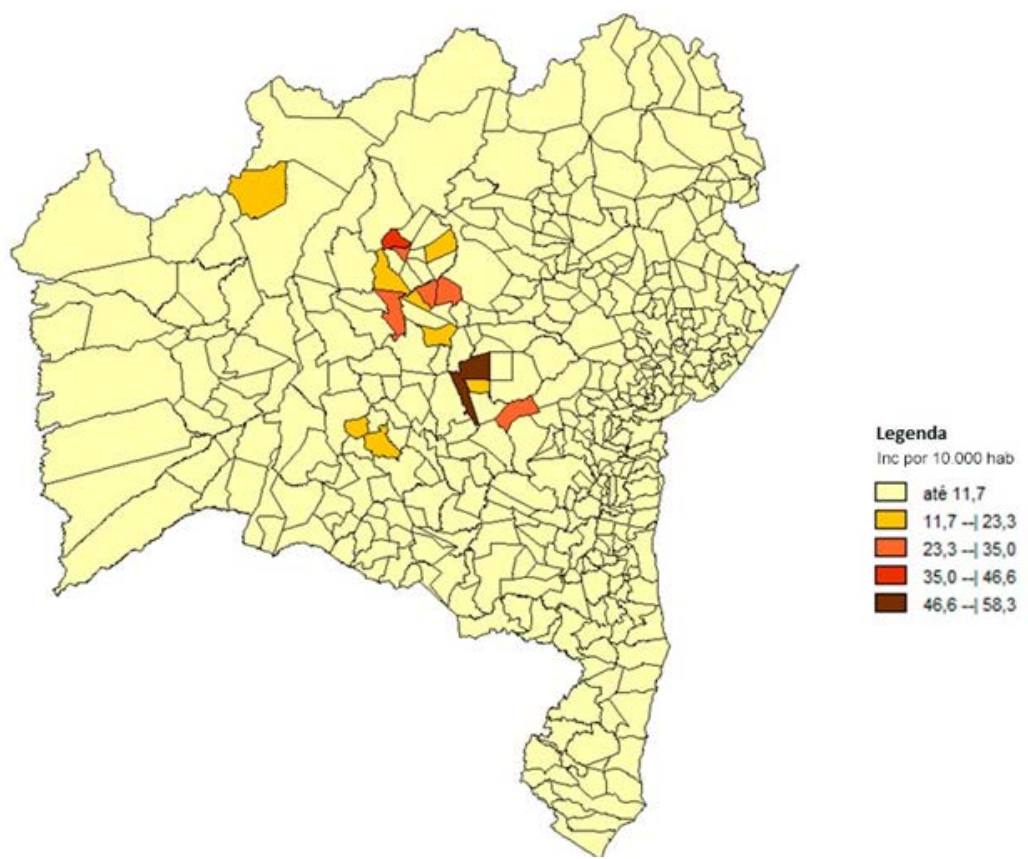

Figura 4. Distribuição dos casos de Leishmaniose Visceral por município e coeficiente de incidência, Bahia, 2015. Fonte: SESAB, 2015.

A taxa de incidência calculada para 2015 e 2016, mostra que o município apresenta uma taxa baixíssima em relação ao demais município do estado (Figura 4).

Conforme a SESAB, em 2015, até março, observa-se um coeficiente de incidência de 33,3\% (0,1/10.000hab), e redução média de $50 \%$ entre os anos de 2014 e 2015, no mesmo período (janeiro a março) para o estado. As maiores incidências foram observadas nos municípios de Andaraí (5,8/10.000 hab), Central (5,5/10.000 hab), Barra do 
Mendes (2,8/10.000 hab) e Marcionílio Souza $(2,7 / 10.000 \mathrm{hab})$.

Embora o estudo não tenha evidenciado casos de LV entre os anos de 2010 a 2014, dados publicados pela SESAB, mostraram que o Município de
Formosa do Rio Preto, Bahia, apresentava situação de risco quanto à ocorrência da doença. Essa classificação segundo o risco de ocorrência pressupõe que o município estava classificado em transmissão moderada (Figura 5).

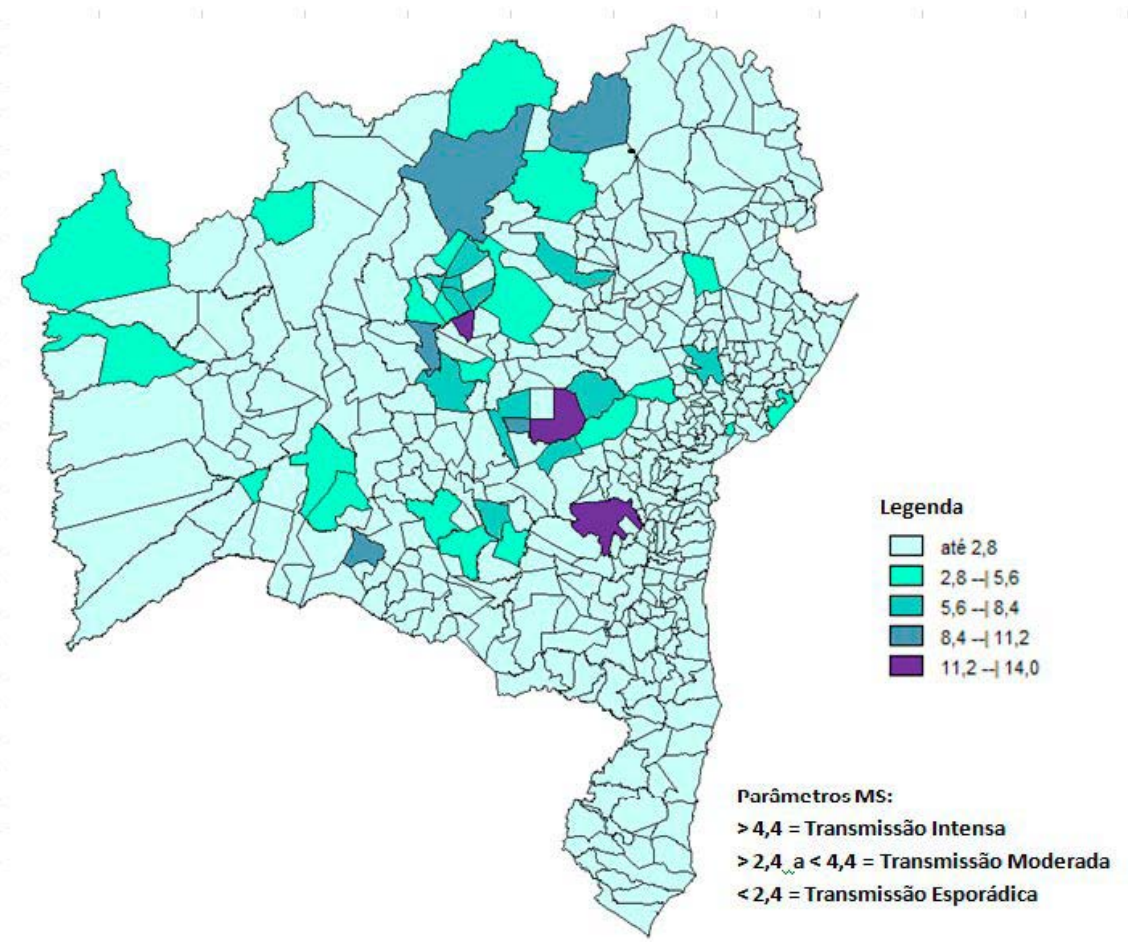

Figura 5. Distribuição dos casos de Leishmaniose Visceral por municípios, segundo classificação de risco, Bahia, 2012-2014. Fonte: SESAB, 2015.

Desta forma, os resultados da taxa de incidência mostram uma associação relativa com a classificação de risco de ocorrência da LV no município feita em 2014 pela Secretaria de Saúde do Estado da Bahia, não saindo da curva de risco de transmissão moderada.

\section{canina \\ Casos de leishmaniose visceral}

Quanto à LV canina, foram identificados 360 casos da doença em cães no município, distribuídos entres os anos de 2013 a 2016 (Figura 6). Para os anos de 2010, 2011 e 2012, não foram confirmados casos de cães infectados com a doença, entretanto foram realizados aproximadamente 75 testes neste período.

No ano de 2013, houve 49 casos de LV canina confirmados, com um aumento significativo para o ano de 2014, quando foram notificados 187 casos, o maior já registrado no município, e 110 foram notificados em 2015 e 14 em 2016. Para o último ano observou-se uma redução brusca dos casos da doença em cães. A ocorrência dos casos da LV em cães pode estar relacionada, sobretudo com o fato destes animais frequentarem ambientes que são propícios ao desenvolvimento do vetor da doença, como ambientes silvestres. 


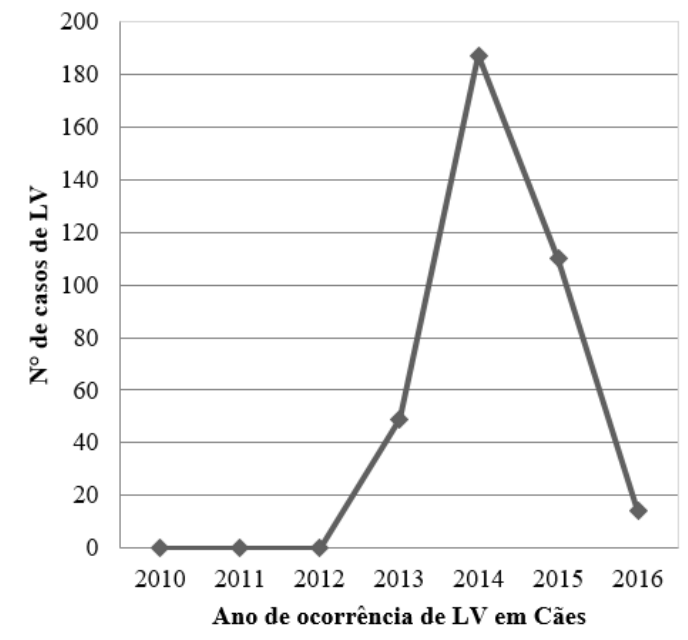

Figura 6. Distribuição dos casos de LV canina no período de 2010 a 2016, no Município de Formosa do Rio Preto, Bahia.

O número expressivo ocorrido no ano de 2014 pode ter relação direta com o fato de que nesse período não houve campanhas de combate aos vetores de transmissão da doença no município. Em contradição com os dados 2014, o ano de 2016 apresentou uma redução significativa dos casos de LV nos cães, visto que a partir da grande ocorrência de casos de 2014, ações voltadas a combater a doença no município passaram a ser desenvolvidas. Nesse sentido, nota-se a importância das campanhas de combate aos vetores de transmissão de doença no município.

\section{Considerações finais}

A distribuição dos casos de leishmaniose visceral entre os anos de 2010 e 2016 apontou que o Município de Formosa do Rio Preto, Estado da Bahia, apresenta um pequeno histórico de casos confirmados da doença, sendo as maiores ocorrências registradas entre os anos de 2014 e 2015. Os maiores casos da LV no município aconteceram em cães com 360 casos confirmados, enquanto que nos humanos foram apenas 8 (oito) casos confirmados distribuídos ao longo dos três principais bairros da cidade. $\mathrm{O}$ pico de ocorrência de casos da doença nos cães foi registrado no ano de 2014 (187 casos), já para os casos em humanos o registro refere-se a 2015 (5 casos). Ambos com redução expressiva em 2016.

A redução dos casos LV canina tem relação direta com o aumento das campanhas de combate ao vetor de transmissão da doença no município, ocorridas entre os aos de 2014 e 2016, o que pode ter correlação com a redução também para os casos de LV em humanos.

A taxa de incidência da doença calculada para população humana apresentou valores baixíssimos se comparados a outros municípios do estado. Além disso, conforme classificação do risco de incidência para o estado, o município de Formosa do Rio Preto apresenta-se em um quadro de transmissão moderada. Situação que garante maior segurança para a população em termos de qualidade de vida.

Deste modo, destaca-se então, que a adoção de medidas preventivas voltadas ao combate de doenças e vetores de incidência principalmente em espaços urbanos, exerce grande influência sobre os problemas de saúde pública na maioria dos municípios brasileiros, podendo reduzir surtos de adoecimento da população residente.

\section{Declaração de conflitos de interesse}

Os autores declaram não haver conflitos de interesse.

\section{Referências}

Almeida, A. B. P. F.; Mendonça, A. J.; Sousa, V.R.F. Prevalência e epidemiologia da leishmaniose visceral em cães e humanos, na Cidade de Cuiabá, Mato Grosso, Brasil. Ciência Rural, v. 40, n. 7, p. 1610-1615, 2010. https://doi.org/10.1590/S010384782010005000102

Barbosa, I. R. Epidemiologia da leishmaniose visceral no Estado do Rio Grande do Norte, Brasil. Revista de Epidemiologia e Controle 
de Infecção, v. 3, n. 1, p. 17-21, 2013. https://doi.org/10.17058/reci.v3i1.3148

Barboza, D. C. P. M.; Gomes Neto, C. M. B.; Leal, D. C.; Bittencourt, D. V. V.; Carneiro, A. J. B.; Souza, B. M. P. S.; Oliveira, L. S.; Julião, F. S.; Souza, V. M. M.; Franke, C. R. Estudo de coorte em áreas de risco para leishmaniose visceral canina, em municípios da Região Metropolitana de Salvador, Bahia, Brasil. Revista Brasileira de Saúde e Produção Animal, v. 7, n. 2, p. 152-163, 2006.

Bonney, K. M. Promoting civic engagement with neglected tropical disease education. Brazilian Journal of Biological Sciences, $\begin{array}{lll}\text { v. } 3, & \text { n. } 5, & \text { p. } 23-26,\end{array}$ https://doi.org/10.21472/bjbs.030502

Brasil. Secretaria de Vigilância e Saúde. Casos confirmados de leishmaniose visceral, Brasil, grandes regiões e unidades federadas: 1990 a 2011. 2012. Disponível em: <http://portalarquivos.saude.gov.br/ images/pdf/2014/agosto/20/LV-Casos.pdf>. Acesso em: 19 dez. 2016.

Calvopina, M.; Armijos, R. X; Hashiguchi, Y. Epidemiology of leishmaniasis in Ecuador: current status of knowledge: a review. Memórias do Instituto Oswaldo Cruz, v. 99, n. 7, p. 663-672, 2004. https://doi.org/ 10.1590/S0074-02762004000700001

Cavalcante, I. J. M.; Vale, M. R. Aspectos epidemiológicos da leishmaniose visceral (calazar) no Ceará no período de 2007 a 2011. Revista Brasileira de Epidemiologia, v. 17, n. 4, p. 911-924, 2014. https://doi.org/ 10.1590/1809-4503201400040010

Correia, J. B. Epidemiology of visceral leishmaniasis in Pernambuco, North-East of Brazil and the use of a Latex Agglutination Test in Urine for its diagnosis. Liverpool: Liverpool School of Tropical Medicine, 1998. (Tese de doutorado).

Costa, J. M. L.; Tadai, M. S.; Netto, E. M.; Vale, K. C.; Lago, E. L.; Marsden, P. D. Procedência de pacientes portadores de leishmaniose tegumentar americana nas áreas endêmicas de Três Braços e Corte de Pedra, Estado da Bahia, Brasil. Revista da Sociedade Brasileira de Medicina Tropical, v. 21, n. 3, p. $145-149,1988$.

Dantas-Torres, F.; Solano-Gallego, L.; Baneth, G.; Ribeiro, V. M.; Cavalcanti, M. P.; Otranto, D. Canine leishmaniosis in the Old and New Worlds: unveiled similarities and differences. Trends in Parasitology, v. 28, n. 12, p. 531-
538, 2012. https://doi.org/10.1016/ j.pt.2012.08.007

Gusmão, J. D.; Brito, P. A.; Leite, M. T. S. Perfil epidemiológico da leishmaniose visceral no Norte de Minas Gerais, Brasil, no período de 2007 a 2011. Revista Baiana de Saúde Pública, v. 38, n. 3, p. 615-624, 2014. https://doi.org/10.5327/Z0100-02332014380300009

IBGE - Insitituto Brasileiro de Geografia e Estatistica. Cidades. Disponível em: <http://www.ibge.gov.br>. Acesso em: 20 dez. 2017.

Leite, A. I.; Araújo, L. B. Leishmaniose visceral: aspectos epidemiológicos relacionados aos óbitos em Mossoró-RN. Revista de Patologia Tropical, v. 42, n. 3, p. 301-308, 2013. https://doi.org/10.5216/ rpt.v42i3.26928

Marques, N. T. A.; Gonçalves, V. M. N.; Almeida, C. B.; Franco, M. L.; Galhardo, J. A. Geoprocessamento aplicado à epidemiologia da leishmaniose visceral. Hygeia, v. 13, n. 26, p. 156-165, 2017. https://doi.org/10.14393/ Hygeia132613

Montalvo, A. M.; Fraga, J.; Monzote, C. L.; Garcia, G.; Fonseca, L. Diagnóstico de la leishmaniasis: de la observación microscópica del parásito a la detección del ADN. Revista Cubana de Medicina Tropical, v. 64, n. 2, p. 108-131, 2012. Disponível em: <http://scielo.sld.cu/pdf/ mtr/v64n2/mtr02212.pdf>. Acesso em: 20 dez. 2017.

OMS - Organização Mundial de Saúde. Essential Leishmaniasis Maps. Disponível em: <http://www.who.int/leishmaniasis/leishma niasis_maps/en/>. Acesso em: 09 out. 2017.

Ortiz, R. C.; Anversa, L. Epidemiologia da leishmaniose visceral em Bauru, São Paulo, no período de 2004 a 2012: um estudo descritivo. Epidemiologia e Serviços de Saúde, $\quad$ v. 24, $\quad$ n. 1, p. 97-104, 2015. https://doi.org/10.5123/S1679-497420150 00100011

Petersen. C. A.; Barr, S. C. Canine leishmaniasis in North America: emerging or newly recognized? Veterinary Clinics of North America: Small Animal Practice, $\begin{array}{lll}\text { v. 39, } & \text { p. } 6, \quad \text { p. } 1065-1074, \quad 2009 .\end{array}$ https://doi.org/10.1016/j.cvsm.2009.06.008 Queiroz, M. J. A.; Alves, J. G. B.; Correia, J. B. Visceral leishmaniasis: clinical and epidemiological features of children in an endemic area. Jornal de Pediatria, v. 80, n. 2, 
p. 141-146, 2004. https://doi.org/10.1590/ S0021-75572004000200012

Rodrigues, A. C. M.; Melo, A. C. F. L.; Júnior, A. D. S.; Franco, S. O.; Rondon, F. C. M.; Bevilaqua, C. M. L. Epidemiologia da leishmaniose visceral no Município de Fortaleza, Ceará. Pesquisa Veterinária Brasileira, v. 37, n. 10, p. 1119-1124, 2017. https://doi.org/10.1590/s0100-736x2017 001000013

SESAB - Secretaria de Saúde do Estado da Bahia. Situação epidemiológica da leishmaniose visceral no Estado da Bahia. Salvador: SUVISA, Ano 3, n. 1, 2015. Disponível em: <http://www.saude.ba. gov.br/wp-content/uploads/2017/11/2015Boletim-de-Leishmaniose-Visceral-n.01.pdf>. Acesso em: 19 dez. 2017.

Silva, F. A.; Fernandes, H. F.; Costa, D. A.; Ramos, E. F. Phlebotominae (Diptera: Psychodidae) na zona urbana do Município de Rio Tinto, Paraíba, Brasil. Revista Brasileira de Gestão Ambiental e Sustentabilidade, $\quad$ v. 4 , n. 8, p. 343-354, 2017. https://doi.org/10.21438/rbgas.040809

Silva, S. M.; Rabelo, P. F. B.; Gontijo, N. F.; Ribeiro, R. R.; Melo, M. N.; Ribeiro, V. M.; Michalick, M. S. M. First report of infection of Lutzomyia longipalpis by Leishmania (Leishmania) infantum from a naturally infected cat of Brazil. Veterinary Parasitology, v. 174 , n. $1 / 2$, p. $150-154$, 2010. https://doi.org/10.1016/j.vetpar. 2010.08.005
Souza, A. P. L.; Jesus, J. R.; Teixeira, M. C. Estudo retrospectivo da epidemiologia da leishmaniose visceral no Rio Grande do Sul. Veterinária em Foco, v. 11, n. 2, p. 112-118, $2014 . \quad$ Disponível em: <http://www.periodicos.ulbra.br/index.php/ veterinaria/article/view/1291/1415>.

Acesso em: 19 dez. 2017.

Waldman, E. A. Vigilância em Saúde Pública. 7.ed. São Paulo: Fundação Peirópolis, 1998.

WHO - World Health Organization. Control of the leishmaniases: report of a meeting of the WHO Expert Committee on the Control of Leishmaniases. Geneva: WHO, 2010. (WHO Technical Report Series, 949). Disponível em: <http://apps.who.int/iris/bitstream/10665/ 44412/1/WHO_TRS_949_eng.pdf >. Acesso em: 19 dez. 2017.

Ximenes, M. F. F. M.; Silva, V. P. M.; Queiroz, P. V. S.; Rego, M. M.; Cortez, A. M.; Batista, L. M. M.; Medeiros, A. S.; Jeronimio, S. M. B. Flebotomíneos (Diptera: Psychodidae) e leishmanioses no Rio Grande do Norte, Nordeste do Brasil: reflexos do ambiente antrópico. Neotropical Entomology, v. 36, n. 1, p. 128-137, 2007. https://doi.org/ 10.1590/S1519-566X2007000100016

Informação da Licença: Este é um artigo Open Access distribuído sob os termos da Licença Creative Commons Attribution, que permite uso irrestrito, distribuição e reprodução em qualquer meio, desde que a obra original seja devidamente citada. 